\title{
Acute Pulmonary Thromboembolism Retrospective Auditory - The Benefits of Clinical Predictive Tools
}

\author{
Bruno Sousa Dos Santos ${ }^{1,2, *}$, Maya Wielert ${ }^{3}$, Ieva Krieva ${ }^{1,2}$, Kathryn Mullan ${ }^{4}$, \\ Sandra Seimane ${ }^{2}$, Aleksejs Visnakovs ${ }^{2}$, Olegs Sabelnikovs ${ }^{1,5}$ \\ ${ }^{1}$ Department of Anesthesiology, Faculty of Medicine, Riga Stradins University, Riga, Latvia \\ ${ }^{2}$ Department of Emergency, Riga East Clinical University Hospital (RECUH), Riga, Latvia \\ ${ }^{3}$ Faculty of Medical, University of Lübeck, Lübeck, Germany \\ ${ }^{4}$ Department of Medicine, Altnagelvin Area Hospital, Derry/Londonderry, Northern Ireland, UK \\ ${ }^{5}$ Department of Anesthesiology, Pauls Stradins Clinical University Hospital, Riga, Latvia
}

Copyright $\mathrm{O} 2019$ by authors, all rights reserved. Authors agree that this article remains permanently open access under the terms of the Creative Commons Attribution License 4.0 International License

\begin{abstract}
Several studies in recent years have identified the importance of developing a more standardized diagnostic approach to patients suspected of acute pulmonary embolism (PE). Still many doctors prefer a theoretical approach to the diagnosis of PE without the use of pre-test clinical predictive tools. Through the process of audit, we collected data available from the files of patients who underwent computer tomography pulmonary angiography (CTPA) and tested this data within the pre-test probability tool "Wells criteria score". Additionally, other important variables that were previously validated as well as those with possible diagnostic value were also analysed, as these may prove to be useful indicators to further develop the pathway towards a more efficient diagnosis of PE. The results within this study were compared to similar studies. With the aim of understanding the possible benefits of a more purposely structured approach, this study ultimately intends to support the development of PE protocols towards a more standardized approach to its diagnosis. Subsequently, it seeks to promote improvement in patient safety and accuracy in diagnosis as well as clinical decision-making. The main results in this study show a clear correlation between Wells criteria score risk levels with an increased predictive likelihood of a positive PE. Dyspnea and chest pain proved to be the most frequent clinical findings outside wells criteria. The age-adjusted D-dimer in this study data demonstrated to be a candidate for further evaluation with great potential for clinical application. Electrocardiogram S1Q3T3 findings displayed a specificity of $92,86 \%$ and positive predictive value $69,70 \%$. The conclusion of this study reveals that a standardized approach that includes Wells criteria together with an age-adjusted D-dimer may avoid unnecessary steps and examinations in diagnosis of $\mathrm{PE}$, namely that of $\mathrm{D}$-dimer tests and CTPA.
\end{abstract}

Keywords Pulmonary Embolism, Thromboembolism, DVT, Wells, D-dimer, Electrocardiogram, Angiography

\section{Introduction}

Epidemiology: Deep vein thrombosis/pulmonary embolism (DVT/PE) is the third most common disease among cardiovascular diseases, and its overall incidence ranges from 1/1000 to 2/1000 per year [1], [2]. In Europe, there is an estimated 684000 DVT cases and $434723 \mathrm{PE}$ cases per annum with the death toll from venous thromboembolism reaching 543454 per annum. The same study estimates a death toll of 370012 in the six European countries which were involved in the study. Of these cases, only $7 \%$ were diagnosed and possibly received appropriate treatment. The remaining $93.0 \%$ were only confirmed postmortem. In effect, they either went undiagnosed and therefore untreated $(59.0 \%)$ or suffered sudden fatal PE (34.0\%) [3], [4].

PE pathogenesis: $\mathrm{PE}$ occurs due to the formation of a thrombus which subsequently travels to the pulmonary arteries. Virchow's triad states the main risk factors of thrombus formation, it consists of: (1) venous stasis or blood flow alterations, (2) vascular endothelial injury and (3) inherited or acquired hypercoagulability state [5], [6].

Clinical presentations in PE: PE can manifest with a wide variety of clinical presentations. A study by P. D. Stein et al. showed the following frequencies of the most common clinical presentations: dyspnea at rest or with exertion was present in $73.0 \%$ of cases, pleuritic pain in $66.0 \%$, calf or thigh pain and/or swelling in $44.0 \%$, cough in $37.0 \%$, orthopnea in $28.0 \%$, wheeze in $21.0 \%$ and hemoptysis was present in $13.0 \%$ of cases [7]. Conversely, some patients exhibit severe clinical presentations such as 
sudden death or shock as well as atypical symptoms, while others remain entirely asymptomatic [7]-[10].

1) Diagnostic approach to PE: Approach to PE is mostly done by theoretical "unstructured" approach and confirmed by CTPA after a positive D-dimer test result is carried out in the emergency department (ED) of Riga East Clinical University Hospital (RECUH). A study done by C. R. Weiss et al. reports that a great percentage of doctors at other centers also prefer an "unstructured" approach for pre-test assessment $(72.5 \%)$. Only $22.9 \%$ reported to use validated predictive tools and/or guidelines. However, 93.0\% were aware of the updated guidelines and $44.2 \%$ reported to use them in practice on a daily basis [11].

2) The challenges of implementing algorithms for the diagnosis of PE have always been present within everyday clinical practice. The use of combined algorithms has proved beneficial. Well validated algorithms often contain: (1) a clinical pre-test probability tool (for instance Wells criteria score), (2) a D-dimer test, and (3) a CTPA [2], [12], [13].

Clinical predictive tools: The pre-test probability tools for the diagnosis of PE have been compared in different studies. Wells criteria score and Geneva score are among the most commonly used tools by physicians and recommended by several guidelines in the approach to the diagnosis of PE [2], [13]. From these pre-test probability tools, Wells criteria is the most frequently used [2]. Based on this information and the caveat that today's doctors are currently more familiar with the Wells criteria, we have selected Wells criteria as the pre-test probability tool to be used in our study.

Wells score criteria (Table 1.): The Wells score consists of one subjective criterion "PE is the first diagnosis, or equally likely" and six objective criteria of varying weight. Different amounts of points are assigned to the positive criteria. The sum total of these points allows patients to be categorized into a three-level pre-test probability/risk: low $(<2$ points); intermedium (2 to 6 points); and high ( $>6$ points). Wells score can also be interpreted as a two-level probability/risk: low ( $\leq 4$ points) and high ( $>4$ points). In this study, we have used the three-level score.

Table 1. The Wells score criteria with respective points for each criterion.

\begin{tabular}{cc}
\hline \multicolumn{2}{c}{ Wells score criteria } \\
\hline Criteria & Points \\
\hline Clinical signs and symptoms of DVT & 3 \\
\hline PE is the first diagnosis, or equally likely & 3 \\
\hline Heart Rate $>$ 100 beats per min & 1.5 \\
\hline $\begin{array}{c}\text { Immobilization } \geq 3 \text { days, or surgery in the previous } 4 \\
\text { weeks }\end{array}$ & 1.5 \\
\hline Previous, objectively diagnosed PE or DVT & 1.5 \\
\hline Hemoptysis & 1 \\
\hline Malignancy with treatment within 6 months or palliative & 1 \\
\hline DVT: Deep Vein Thrombosis; PE: Pulmonary Embolism. \\
\hline
\end{tabular}

$D$-dimer test: The use of D-dimer levels adjusted to the patients' age in patients $\geq 50$ years old, also known as "age-adjusted D-dimer", has been thought to be beneficial by means of increasing specificity without effecting sensitivity. It demonstrated sensitivities of $>97 \%$ and higher specificities when compared to the standard cut-off value of a standard D-dimer test $(0.50 \mathrm{mcg} / \mathrm{mL})$. The D-dimer test has a tendency to be elevated within this age group ( $\geq 50$ years old) due to a natural increase in fibrinogen concentration, known and unknown malignancies and age related increase in inflammation, among other reasons [14]. Note that this approach is mainly accepted for hemodynamic stable patients at a low and/or intermediate risk [2], [13], [15], [16].

Electrocardiography (ECG): In the presence of $\mathrm{PE}$ diagnosis, $\mathrm{T}$-wave inversion, $\mathrm{T}$-wave flattening, sinus tachycardia and ST-segment changes are among the most common acute changes on ECG when compared to the previous ECGs [17]. Besides these cited findings, ECG changes that indicate Right Ventricular Dysfunction (RVD) including S-wave on lead 1, Q-wave on lead 3 and $\mathrm{T}$ wave-inversion on lead 3 (S1Q3T3) are also considered at RECUH when suspicion of PE.

\section{Chapters}

\subsection{Aims of the Research}

1) This study intends not only to contribute to a more standardized diagnostic approach to suspected PE in the studied center, but also to put forth results to the greater scientific community with the hope of informing future studies in this clinical field.

2) Study's hypothesis: Reduction of excessive examinations and improvement in patients' safety can be achieved by including a clinical predictive tool (in this study Wells criteria) and/or an age-adjusted D-dimer test.

3) Additionally, we would like to evaluate the benefits of ECG findings, as the ECG is one of the most frequently used diagnostic tests in the emergency settings when a patient presents with chest related symptoms.

\subsection{Tasks}

1) To analyse the data of patients who underwent CTPA for suspected PE from the 2014 archive at Riga East Clinical University Hospital, Riga, Latvia.

2) To compare the statistical analysis of potential relevant data in the diagnosis of PE with previous published studies and clinical guidelines.

3) To discuss possible benefits of using international recommended clinical predictive scores (Wells criteria in this study) for the pre-test probability of PE, age-adjusted D-dimer and the ECG S1Q3T3 findings. 


\section{Methods}

\subsection{Sample}

This is a retrospective study done with data collected from patients that underwent CTPA for suspected PE at the emergency department (ED) of RECUH from January to September of 2014.

\subsection{Flowchart Data}

220 patients out of 249 were included in this study. 29 patients were excluded due to incomplete or unclear data.

\subsection{Clinical Presentations}

The most common clinical presentations were divided into positive and negative PE groups, the relative frequencies and odds ratio (OR) were calculated, as well as its significance between the groups.

For the clinical presentations of blood pressure and arterial oxygenation saturation $\%(\mathrm{SpO} 2)$ the median/mean values and its significance between positive and negative PE groups were calculated.

\subsection{Wells Criteria Score}

First, for each patient included in this study the pre-test probability (risk levels) by the Wells criteria risk score was calculated. The Wells criteria score is represented in Table 1 and its risk levels described on page 3 .

Second, the patients were stratified according to their CTPA result (positive/negative for PE) and their risk level according to the Wells criteria. For each group, the relative frequency of PE was calculated and the correlation between Wells risk levels and the likelihood ratio of a positive result for PE was analyzed.

\subsection{D-dimer / Age-adjusted D-dimer}

The standard threshold for the D-dimer test is set at 0.05 $\mathrm{mcg} / \mathrm{mL}$ regardless of the patient's age. To assess the individual threshold for age-adjusted D-dimer, a calculation was done for each patient of 50 years of age or above. The age-adjusted D-dimer test threshold was calculated using the following formula [16]:

Formula 1

Age-adjusted D-dimer test: (1) Age x $0.010=\mathrm{mcg} / \mathrm{mL}$

Individual D-dimer results were statically analyzed and stratified by the Wells criteria score risk levels. The correlations between D-dimer values and patients of 50 years of age or older for positive and negative PE groups were evaluated.

\subsection{Electrocardiography S1Q3T3 Finding}

The predictive value of S1Q3T3 findings on a ECG as an indicator for RVD in PE was estimated for this population.

\subsection{Statistical Analysis}

Categorical data was compared by the Chi-square test. Continuous data was tested for "skewness" by Shapiro-Wilk test. Mann-Whitney U test was applied to compare means/medians in continuous skewed variables. Spearman's correlation coefficient was used for continuous skewed variables. Statistical significances were calculated at $\mathrm{p} \leq 0.05$, and all data analyze was performed using the software SPSS by IBM, version 23 .

\section{Results, Interpretation, Discussion}

\subsection{Demographic Data}

From a total of 220 CTPA validated cases that are included in this study, $100(45.5 \%)$ cases are male and 120 $(54.5 \%)$ cases are female. The mean age (Standard deviation [SD]) for the positive PE cases was $77.88( \pm 9.11)$ for females and $64.46( \pm 16.83)$ for males. $71 / 220(32.3 \%)$ of cases proved to be positive for PE in CTPA while $149 / 220(67.7 \%)$ of cases were negative.

\subsection{Clinical Presentations Results, Its Significance and Discussion}

Table 2 shows the relative frequencies of the most common clinical presentations within positive and negative PE groups. The included symptoms and signs (clinical presentations) on this study were identified as the most common triggers for patients seeking medical care and/or the likelihood of being admitted to the ED.

Dyspnea proved to be the most frequent clinical presentation for both groups. In the positive PE group, dyspnea showed a relative frequency of $90.1 \%$ compared to the negative PE group (63.7\%), odds ratio [CI 95\%] = 5.21 [2.23-12.19], with the statistical significance between the groups being $\mathrm{p}<0.001$.

Previous studies showed similar results. Miniati et al. reports sudden onset of dyspnea present in $78 \%$ of cases with positive $\mathrm{PE}(\mathrm{n}=281$; 74-82 [95\% CI]) [18]. Stein et al. describe $79 \%$ of cases having dyspnea at rest or exertion [7]. In both studies dyspnea was the most frequent symptom on admission. This corresponds with our results where dyspnea represented the most frequent symptom in cases of positive PE independently of the different dyspnea forms. Thus, it can be denoted that dyspnea plays an important role in identifying patients with possible PE in clinical practice. 
Table 2. Clinical presentations relative frequencies association to positive and negative PE by CTPA results.

\begin{tabular}{|c|c|c|c|c|}
\hline Clinical presentation & Positive PE by CTPA & Negative PE by CTPA & $O R[95 \% \mathrm{CI}]$ & $\begin{array}{c}p \\
\text { Value } \\
\left(\mathrm{X}^{2}\right) \\
\end{array}$ \\
\hline Dyspnea & $64 / 71(90.1)$ & $93 / 146(63.7)$ & $5.21[2.23-12.19]$ & $<.001$ \\
\hline Tachypnea ( $\geq 20$ times per min) & $34 / 67(50.7)$ & $50 / 137(36.5)$ & $1.79[0.99-3.24]$ & .052 \\
\hline Non-specific chest pain & $32 / 70(45.7)$ & $45 / 146(30.8)$ & $1.89[1.05-3.40]$ & .032 \\
\hline Syncope & $13 / 71(18.3)$ & $40 / 149(26.8)$ & $0.61[0.30-1.23]$ & .116 \\
\hline Cough & 12/71 (16.9) & $20 / 148(13.5)$ & $1.30[0.60-2.84]$ & .506 \\
\hline Anxiety & $37 / 71(52.1)$ & $98 / 147(66.7)$ & $0.54[0.31-0.97]$ & .038 \\
\hline Breathing related chest pain & 13/71 (18.3) & $11 / 149(7.4)$ & $2.81[1.19-6.64]$ & $* .020$ \\
\hline Body Temperature $>37.5^{\circ} \mathrm{C}$ & $5 / 19(26.3)$ & $14 / 19(73.7)$ & $0.95[0.30-2.97]$ & $* .000$ \\
\hline ECG atrial fibrillation & 21/71 (30.0) & $48 / 144(33.3)$ & $0.86[0.46-1.59]$ & .625 \\
\hline Hypotension & $15 / 37(40.5)$ & $22 / 37(59.5)$ & $1.57[0.76-3.26]$ & .220 \\
\hline Hypertension & $26 / 93(28.0)$ & $67 / 93(72.0)$ & $0.72[0.40-1.30]$ & .307 \\
\hline Clinical presentation & $\begin{array}{c}\text { Median } \\
\text { "Mean } \pm \text { SD" } \\
\text { (Range) } \\
n=70 \\
\end{array}$ & $\begin{array}{c}\text { Median } \\
\text { "Mean } \pm \text { SD" } \\
\text { (Range) } \\
n=148 \\
\end{array}$ & $\mathrm{U}$ & $\begin{array}{c}P \\
\text { value }\end{array}$ \\
\hline Systolic blood pressure in $\mathrm{mmHg}$ & $\begin{array}{c}131.19 \\
" 129.19 \pm 29.72 " \\
(40-180) \\
\end{array}$ & $\begin{array}{c}138.00 \\
“ 137.90 \pm 32.03 " \\
(67-250) \\
\end{array}$ & 4538.00 & .140 \\
\hline Diastolic blood pressure in $\mathrm{mmHg}$ & $\begin{array}{c}79.50 \\
\text { “74.80 } \pm 16.34 " \\
(20-108) \\
\end{array}$ & $\begin{array}{c}80.00 \\
\text { “76.75 } \pm 18.41 " \\
(25-126) \\
\end{array}$ & 4909.50 & .533 \\
\hline Mean average blood pressure in $\mathrm{mmHg}$ & $\begin{array}{c}96.67 \\
\text { “92.93 } \pm 19.70 ” \\
(26.67-126.67)\end{array}$ & $\begin{array}{c}98.50 \\
\text { “97.13 } \pm 21.51 ” \\
(41.00-162.67)\end{array}$ & 4672.50 & .243 \\
\hline Clinical presentation & $\begin{array}{c}\text { Median } \\
\text { "Mean } \pm \text { SD" } \\
\text { (Range) } \\
n=69\end{array}$ & $\begin{array}{c}\text { Median } \\
\text { "Mean } \pm \text { SD" } \\
\text { (Range) } \\
n=145\end{array}$ & $\mathrm{U}$ & $\begin{array}{c}p \\
\text { value }\end{array}$ \\
\hline Arterial oxygen saturation in \% & $\begin{array}{c}94.00 \\
\text { "92.86 } \pm 4.45 " \\
(99-78) \\
\end{array}$ & $\begin{array}{c}95.00 \\
\text { “93.82 } \pm 5.05 " \\
(71-100) \\
\end{array}$ & 4009.00 & .018 \\
\hline \multicolumn{5}{|c|}{$\mathrm{X}^{2}$ : Chi-square test; * Fisher's Exact Test (Significance 2-sided); U: Mann Whitney U test; } \\
\hline $\begin{array}{l}\text { PE: Acute Pulmonary Thromboembolisr } \\
\text { Pulmonary Embolism. SD: Standard dev }\end{array}$ & $\begin{array}{l}\text { A: Computer Tomog } \\
\text { df: degrees of freedo }\end{array}$ & Pulmonary Angiogr & T: Deep Vein 7 & PE: \\
\hline
\end{tabular}

Table 2 shows the most common clinical presentations for patients with $\mathrm{PE}$ and their relative frequencies for positive and negative PE groups, statistical significance, and odds ratio between the groups.4.3. Wells Score Criteria Results, Its Significance and Discussion.

Breathing related chest pain/pleuritic chest pain was present with a significant relative frequency of $18.3 \%$ in positive $\mathrm{PE}$ cases when compared to the negative $\mathrm{PE}$ cases $(7.4 \%)$, odds ratio [CI 95\%] $=2.81[1.19-6.64]$, with the statistical significance between the groups being $p=0.020$ (Fischer's Exact Test).

Similarly, non-specific chest pain is significantly more frequent in the positive PE cases (45.7\%) than in the negative cases of PE (30.8\%), odds ratio [CI 95\%] $=1.89$ [1.05-3.40], with the statistical significance between the groups being $p=0.032$. Chest pain is reported by Miniati et al. in $39 \%$ of the cases with positive PE $(n=140 ; 34-44$
[95\% CI]) and by Stein et al. to be present in $47 \%$ (pleuritic chest pain) and 17\% (non-pleuritic) [7], [18]. When comparing the data to this study, it shows that the symptom of chest pain is of statistical and clinical significance and one of the main symptoms in patients with PE. Moreover, it is imperative to highlight that non-specific chest pain shows the highest frequencies and statistical significance in our study. However, chest pain in its generalised form is among the leading symptoms for patients with positive PE, and it is the second most frequently reported symptom after dyspnea.

Tachypnea ( $\geq 20$ breaths per minute) occurred in $50.7 \%$ of patients with $\mathrm{PE}$ and in $36.5 \%$ of patients with a negative CTPA result, odds ratio [CI 95\%] $=1.79$ [0.99-3.24], with the statistical significance between the groups being $\mathrm{p}=$ 0.052 .

Syncope showed a relative frequently of $18.3 \%$ for the 
positive PE group and of $26.8 \%$ for the negative PE group, odds ratio $[$ CI 95\%] $=0.61[0.30-1.23]$, with the statistical significance between the groups being $\mathrm{p}=0.116$.

$16.9 \%$ of patients with PE complained of cough while $26.8 \%$ without $\mathrm{PE}$ also had cough, odds ratio [CI 95\%] = $1.30[0.60-2.84]$, with the statistical significance between the groups being $\mathrm{p}=0.506$.

Anxiety was present with a relative frequently of $52.1 \%$ in $\mathrm{PE}$ cases and $66.7 \%$ for the negative PE group, odds ratio $[$ CI 95\%] $=0.54[0.31-0.97]$, with the statistical significance between the groups being $\mathrm{p}=0.038$.

$26.3 \%$ of positive $\mathrm{PE}$ cases and in $73.7 \%$ of negative $\mathrm{PE}$ cases presented with a body temperature $>37^{\circ} \mathrm{C}$, odds ratio [CI 95\%] $=0.95[0.30-2.97]$, with the statistical significance between the groups being $\mathrm{p}=0.000$ (Fischer's Exact Test).

Patients with PE showed hypotension in $40.5 \%$ of the cases. In patients without $\mathrm{PE}$ hypotension was present with a relative frequency of $59.5 \%$, odds ratio [CI 95\%] $=1.57$ [0.76-3.26], with the statistical significance between the groups being $\mathrm{p}=0.220$.

Conversely, hypertension occurred with a relative frequently of $28.0 \%$ for the positive cases of $\mathrm{PE}$ and of $72.0 \%$ for negative $\mathrm{PE}$ cases, odds ratio [CI 95\%] $=0.72$ [0.42-1.30], with the statistical significance between the groups being $\mathrm{p}=0.307$.

Systolic blood pressure of positive PE cases exhibited a median value of $131.19 \mathrm{mmHg}$ and mean value of $129.19 \pm$ $29.72[$ Mean $\pm \mathrm{SD}$ ] with a range of $40.00-180.00 \mathrm{mmHg}$. The negative group for PE showed a median value of $138.00 \mathrm{mmHg}$ and a mean value of $137.90 \pm 32.03$ [Mean \pm SD] with a range of $67.00-250.00 \mathrm{mmHg}$. Mann Whitney $\mathrm{U}$ test showed for systolic blood pressure a result of $\mathrm{U}=$ 4538.00 , with the statistical significance between the groups being $\mathrm{p}=0.140$.

Diastolic blood pressure showed a median value of $79.50 \mathrm{mmHg}$ and a mean value of $74.80 \pm 16.34$ [Mean \pm SD] for the positive cases of PE with a range of 20.00 $108.00 \mathrm{mmHg}$. The negative group for PE showed a median value of $80.00 \mathrm{mmHg}$ and a mean value of $76.75 \pm$ 18.41 [Mean \pm SD] with a range of $25.00-126.00 \mathrm{mmHg}$. Mann Whitney U test for systolic blood pressure showed a result of $U=4909.50$, with the statistical significance between the groups being $\mathrm{p}=0.533$.

Altogether, the mean average blood pressure had a median value of $96.67 \mathrm{mmHg}$ and a mean value of $92.93 \pm$ 19.74 [Mean \pm SD] with a range of $26.67-126.67 \mathrm{mmHg}$ for positive PE cases. The negative group for PE showed a median value of $98.50 \mathrm{mmHg}$ and a mean value of $97.13 \pm$ 21.51 [Mean \pm SD] with a range of $41.00-162.67 \mathrm{mmHg}$. Mann Whitney U test showed for systolic blood pressure clinical presentation a result of $U=4672.50$, with the statistical significance between the groups being $\mathrm{p}=0.243$.

The statistical analysis of arterial oxygen saturation \% (SpO2) proved a significant difference between the medians of the positive and negative PE groups, with the positive group for $\mathrm{PE}$ having a lower median when compared to the negative group. In numbers, the positive group presented a median of $94.00 \% \mathrm{SpO} 2$ and a mean value of $92.86 \pm 4.45$ [Mean $\pm \mathrm{SD}$ ] with the range of 99.00 $-78.00 \% \mathrm{SpO} 2$. Conversely, the median for the negative $\mathrm{PE}$ group was $95.00 \% \mathrm{SpO} 2$ and the mean value $93.82 \pm$ 5.05 [Mean \pm SD] with the range of $71.00-100.00 \%$ SpO2. Mann Whitney $U$ test was performed for arterial oxygen saturation with a result of $U=4009.00$, showing the following statistical significance between the groups $\mathrm{p}=$ 0.018 .

Kline et al. reported arterial oxygen saturation (\%) and in-hospital complications for patients with PE as follows: for $\mathrm{SpO} 2<95 \%$ nine out of ten $(90 \%)$ patients had in-hospital complications and for $\mathrm{SpO} 2 \geq 95 \% 31$ out of 86 (36.05\%) patients had in-hospital complications. Mortality for patients with $\mathrm{SpO} 2<95 \%$ was $20 \%$ within 30 days, compared to only $2 \%$ for patients with $\mathrm{SpO} 2 \geq 95 \%$ [19].

Table 3 shows the "Wells criteria relative frequencies and comparison between positive and negative PE for the studied population".

Table 3. Wells criteria calculation relative frequencies results evaluation between positive and negative PE by CTPA.

\begin{tabular}{lcccc}
\hline Wells score criteria & $\begin{array}{c}\text { Positive PE by } \\
\text { CTPA } \\
n=71\end{array}$ & $\begin{array}{c}\text { Negative PE by } \\
\text { CTPA } \\
n=149\end{array}$ & OR [CI 95\%] & $\begin{array}{c}p \text { value } \\
\left(X^{2}\right)\end{array}$ \\
\hline Clinical signs and symptoms of DVT. $n(\%)$ & $36(50.7)$ & $34(22.8)$ & $3.48[1.91-6.35]$ & $<.001$ \\
\hline PE is 1st diagnosis or equally likely. $n(\%)$ & $68(95.8)$ & $122(81.9)$ & $5.02[1.47-17.15]$ & $* .005$ \\
\hline Heart Rate > 100/min. $n$ (\%) & $31(43.7)$ & $49(32.9)$ & $1.59[0.89-2.83]$ & .120 \\
\hline $\begin{array}{c}\text { Immobilization } \geq 3 \text { days, or surgery in the previous } 4 \\
\text { weeks. } n \text { (\%) }\end{array}$ & $17(23.9)$ & $22(14.8)$ & $1.82[0.90-3.69]$ & .096 \\
\hline Previous, objectively diagnosed PE or DVT. $n$ (\%) & $22(31.0)$ & $15(10.1)$ & $4.01[1.93-8.35]$ & $<.001$ \\
\hline Hemoptysis. $n$ (\%) & $4(5.6)$ & $5(3.4)$ & $1.72[0.45-6.61]$ & $* .474$ \\
\hline $\begin{array}{l}\text { Malignancy with treatment within 6 months or palliative. } \\
n \text { (\%) }\end{array}$ & $10(14.1)$ & $8(5.4)$ & $2.89[1.09-7.68]$ & .027 \\
\hline * Fisher's Exact Test (Exact Significance 2-sided); & & & \\
\hline $\begin{array}{l}\text { PE: Acute Pulmonary Thromboembolism; CTPA: Computer Tomography Pulmonary Angiography; DVT: Deep Vein Thrombosis; PE: } \\
\text { Pulmonary Embolism. }\end{array}$
\end{tabular}


Table 3 Shows the Wells score criteria "clinical presentations", their relative frequencies for positive and negative PE groups, statistical significance, and odds ratio between the groups.

For the criterion "Clinical signs and symptoms of DVT" the positive cases of PE were significantly more frequent $(50.7 \%)$ than the negative cases of PE $(22.8 \%)$, odds ratio [CI 95\%] $=3.48$ [1.91-6.35], with the statistical significance between the groups being $p<0.001$.

For the criterion "Previous, objectively diagnosed $\mathrm{PE}$ or DVT" the positive cases of PE were significantly more frequent $(31.0 \%)$ than the negative cases of PE $(10.1 \%)$, odds ratio [CI 95\%] $=4.01[1.93-8.35]$, with the statistical significance between the groups being $p<0.001$.

Within Wells score criteria for our studied population the criteria that showed greater statistical significance were "Clinical signs and symptoms of DVT" and "Previous, objectively diagnosed PE or DVT".

"Clinical signs and symptoms of DVT" had a relative frequency of $50.7 \%$. This frequency proved to be similar to other previous reported studied data, for instance as by Posadas-Martínez et al., where the relative frequency for "Clinical signs and symptoms of DVT" was reported to be $62.3 \%$ for positive PE patients and $2 \%$ for negative PE patients.

The same study reported "Previous, objectively diagnosed PE or DVT" with a relative frequency of $13.1 \%$ for positive PE patients and $4.9 \%$ for negative PE patients. Additionally, Posadas-Martínez et al., reported "Immobilization $\geq 3$ days, or surgery in the previous 4 weeks" with the highest relative frequency of $74.3 \%$ for positive PE patients and $62 \%$ for negative PE patients [20]. Comparable relative frequencies are present for our studied population.

For the criterion "Malignancy with treatment within 6 months or palliative" the positive cases of PE were significantly more frequent $(14.1 \%)$ than the negative cases of PE $(5.4 \%)$, odds ratio [CI 95\%] = 2.89 [1.09-7.68], with the statistical significance between the groups being $p=$ 0.027. This criterion has been suggested to be relevant, especially due to the strong evidence of the pathophysiologic - hypercoagulability states in cancer patients. PE accounts for the second most common cause of death in cancer patients. Undergoing chemotherapy also increases the risk of thrombosis [21].

For the criterion "PE is 1st diagnosis or equally likely" the PE positive cases were significantly more frequent $(95.8 \%)$ than the negative cases of PE $(81.9 \%)$, odds ratio [CI 95\%] $=5.02$ [1.47-17.15], with statistical significance between the groups $p=0.005$ (Fischer's Exact Test).

"PE is 1st diagnosis or equally likely" is the subjective criterion that has been most criticized. On the other hand, in a multivariable regression analysis it also has shown to be one of the most important criteria of the Wells score.
The physician's decisions to validate this criterion have been influenced by the presence of one or more criteria of the Wells score [22].

We believed that in our data the criterion "PE is 1 st diagnosis or equally likely" in general does not derive from the physician being influenced by the presence of other criteria in the score tool, but instead from the clinical suspicion of a positive PE diagnosis to be likely present. This is supported by the fact that most of the physicians in the studied center were not using a score system but an unstructured theoretical approach to the diagnosis of PE instead. Additionally, all our patients underwent CTPA with query PE. However, we could not say if this was performed before or after a D-dimer test. Nevertheless, a D-dimer test was done at RECUH for most cases suspicious of PE.

The criterion that did not reach statistical significance was that of hemoptysis; despite this, its clinical significance remains, especially given that its presence may be an indicator of a more severe pulmonary thromboembolism. Hemoptysis itself with or without the presence of a positive PE can be a life-threatening complication [2], [23].

We found hemoptysis with a relative frequency of $5.6 \%$ in patients with a positive and $3.4 \%$ with a negative CTPA result for PE, odds ratio [CI 95\%] $=1.72$ [0.45-6.61], with the statistical significance between the groups being $p<$ 0.474 (Fischer's Exact Test).

A heart rate $>100 / \mathrm{min}$ was reported in $43.7 \%$ of positive $\mathrm{PE}$ cases and in $32.9 \%$ of patients negative to $\mathrm{PE}$, odds ratio $[$ CI 95\%] $=1.59[0.89-2.83]$, with the statistical significance between the groups being $p<0.120$.

The criterion "Immobilization $\geq 3$ days or surgery in the previous 4 weeks" was present in $23.9 \%$ positive to $\mathrm{PE}$ and in $14.8 \%$ negative to $\mathrm{PE}$, odds ratio [CI 95\%] $=1.82$ [0.903.69], with the statistical significance between the groups being $p<0.096$.

Table 4 shows the Wells criteria risk score calculation by risk levels and the respective $\mathrm{PE}$ results. This assessment gave us the following results: As the risk level increases the predictive likelihood of a positive PE also increases, with the statistical significant likelihood ratio being $(2 \mathrm{df}, n=220)=27.154, p<0.001$.

Wells criteria and other similar clinical predictive tools have been widely validated and their performances have been compared. Most studies indicate similar results between the different clinical predictive tools in the pre-test probability calculation for patients suspected of PE. Their benefits have been reported and recommended by international guidelines [2], [13], [22], [24]. Likewise, our data shows the higher the Wells criteria risk level that a patient is assigned to, the higher the likelihood of a positive $\mathrm{PE}$ result. 
Table 4. Well's criteria risk levels calculation for positive and negative PE by CTPA results.

\begin{tabular}{lccc}
\hline $\begin{array}{l}\text { Wells criteria } \\
\text { risk levels }\end{array}$ & $\begin{array}{c}\text { Cases per } \\
\text { risk. } n\end{array}$ & $\begin{array}{c}\text { Positive PE by } \\
\text { CTPA. } n(\%)\end{array}$ & $\begin{array}{c}\text { Negative PE by } \\
\text { CTPA. } n(\%)\end{array}$ \\
\hline Low risk & 22 & $1(4.5)$ & $21(95.5)$ \\
\hline $\begin{array}{l}\text { Intermediate } \\
\text { risk }\end{array}$ & 139 & $36(25.9)$ & $103(74.1)$ \\
\hline High risk & 59 & $34(57.6)$ & $25(42.4)$ \\
\hline $\begin{array}{l}N=220 ; \text { PE: Acute Pulmonary Thromboembolism; } \\
\text { CTPA: Computer Tomography Pulmonary Angiography. }\end{array}$ \\
\hline
\end{tabular}

Table 4 Shows the Well's criteria risk levels stratification of the 220 patients, risk levels relative frequencies stratified by for positive and negative PE by CTPA results.

\subsection{D-dimers Results Evaluation, Its Significance and Discussion}

\subsubsection{D-dimer Demographic Results}

D-dimer results with a cutoff value of $0.50 \mathrm{mcg} / \mathrm{mL}$ were elevated in 209/220 (95\%) of the cases and not elevated in $2 / 220(0.9 \%)$ of the cases. In $9 / 220(4.1 \%)$ cases D-dimer were not taken.

\subsubsection{D-dimer Results Stratified by Positive and Negative PE Groups}

For the positive cases of PE, D-dimer had a minimum value of $1.35 \mathrm{mcg} / \mathrm{mL}$, a maximum value of $36.72 \mathrm{mcg} / \mathrm{mL}$ a mean value of $13.80 \pm 11.3$ [Mean $\pm \mathrm{SD}] \mathrm{mcg} / \mathrm{mL}$, and a median value of $9.25 \mathrm{mcg} / \mathrm{mL}$.

For negative cases of PE, D-dimer had a minimum value of $0.17 \mathrm{mcg} / \mathrm{mL}$, a maximum value of $35.13 \mathrm{mcg} / \mathrm{mL}$, a mean value of $7.58 \pm 8.22$ [Mean $\pm \mathrm{SD}$ ] $\mathrm{mcg} / \mathrm{mL}$, and a median value of $4.05 \mathrm{mcg} / \mathrm{mL}$. These results can be seen in Table 5 .

\subsubsection{D-dimer Results Stratified by Wells Criteria Risk Score Levels and PE Groups}

On Table 5, D-dimer test results were stratified according to Wells criteria score risk levels with the following results:

For the low risk level 22/22 (100\%) patients had elevated D-dimer. From these one case $(4.5 \%)$ was positive for PE and 21 (95.5\%) of cases were negative for PE.

For the intermediate risk level in 132/139 (95\%) patients D-dimer were elevated, in 2/139 (1.4\%) D-dimer were below the standard threshold, and in 5/139 (3.6\%) D-dimer were not taken. From all 139 cases in the intermediate risk level $36(25.9 \%)$ were positive and $103(74.1 \%)$ were negative for $\mathrm{PE}$.

For the high risk level, D-dimer were elevated in 55/59 $(93.2 \%)$ of the patients and not taken in $4 / 59(6.8 \%)$ of the patients. From these 59 cases, 34 (57.6\%) were positive for $\mathrm{PE}$ and 25 (42.4\%) of cases were negative for PE.

\subsubsection{D-dimer Values Stratified by Wells Criteria Risk Groups based on CTPA Results for PE}

For the low risk group based on Wells criteria, CTPA results presented $21 / 22$ cases negative to PE confirmed by CTPA, from these a minimum D-dimers value of 0.63 $\mathrm{mcg} / \mathrm{mL}$ and a maximum D-dimer value of $35.13 \mathrm{mcg} / \mathrm{mL}$ with a range of 34.50 and a mean value of $9.72 \pm 9.39$ $[$ Mean $\pm \mathrm{SD}$ ]. For the positive PE cases, $1 / 22$ positive PE case confirmed by CTPA with a D-dimer value of 14.99 $\mathrm{mcg} / \mathrm{mL}$.

For the medium-risk group based on Wells criteria, CTPA results presented 99/134 cases negative to PE confirmed by CTPA, from these a minimum D-dimer value of $0.17 \mathrm{mcg} / \mathrm{mL}$ and a maximum D-dimer value of 35.13 $\mathrm{mcg} / \mathrm{mL}$ with a range of 34.96 and a mean value of $7.74 \pm$ 8.55 [Mean $\pm \mathrm{SD}$ ]. For the positive PE cases, 35/134 positive PE case confirmed by CTPA, from these a minimum $\mathrm{D}$-dimer value of $1.35 \mathrm{mcg} / \mathrm{mL}$ and a maximum D-dimer value of $36.72 \mathrm{mcg} / \mathrm{mL}$ with a range of 35.37 and a mean value of $14.99 \pm 12.00$ [Mean \pm SD].

For the high-risk group based on Wells criteria, CTPA results presented 24/55 cases negative to PE confirmed by CTPA, from these a minimum D-dimer value of 1.01 $\mathrm{mcg} / \mathrm{mL}$ and a maximum D-dimer value of $15.51 \mathrm{mcg} / \mathrm{mL}$ with a range of 14.50 and a mean value of $5.03 \pm 4.50$ $[$ Mean $\pm \mathrm{SD}$ ]. For the positive PE cases, $31 / 55$ positive $\mathrm{PE}$ case confirmed by CTPA, from these a minimum D-dimer value of $2.01 \mathrm{mcg} / \mathrm{mL}$ and a maximum $\mathrm{D}$-dimer value of $35.13 \mathrm{mcg} / \mathrm{mL}$ with a range of 33.12 and a mean value of $12.43 \pm 10.03$ [Mean $\pm \mathrm{SD}]$.

Table 5. D-dimer values by Wells criteria risk groups based on CTPA results for PE.

\begin{tabular}{cccccc}
\hline & \multicolumn{4}{c}{ D-dimer Results $(\mathrm{mcg} / \mathrm{mL})$} \\
\cline { 2 - 6 } & $\begin{array}{c}\text { CTPA } \\
\text { results }(n)\end{array}$ & $\underline{\text { Min }}$ & Max & $\begin{array}{c}\text { Mean } \pm \\
\text { SD }\end{array}$ & Range \\
\hline \multirow{4}{*}{ Low Risk } & $\begin{array}{c}\text { Negative PE } \\
(n=21)\end{array}$ & 0.63 & $\begin{array}{c}35.1 \\
3\end{array}$ & $\begin{array}{c}9.72 \pm \\
9.39\end{array}$ & 34.50 \\
\cline { 2 - 6 } & $\begin{array}{c}\text { Positive PE } \\
(n=1)\end{array}$ & 14.9 & 14.9 & $14.99 \pm$ & 0 \\
Medium & 9 & 9 & 0 & 0 \\
Risk & $\begin{array}{c}\text { Negative PE } \\
(n=99)\end{array}$ & 0.17 & $\begin{array}{c}35.1 \\
3\end{array}$ & $\begin{array}{c}7.74 \pm \\
8.55\end{array}$ & 34.96 \\
\cline { 2 - 6 } & $\begin{array}{c}\text { Positive PE } \\
(n=35)\end{array}$ & 1.35 & $\begin{array}{c}36.7 \\
2\end{array}$ & $\begin{array}{c}14.99 \pm \\
12.00\end{array}$ & 35.37 \\
\hline High Risk & $\begin{array}{c}\text { Negative PE } \\
(n=24)\end{array}$ & 1.01 & $\begin{array}{c}15.5 \\
1\end{array}$ & $\begin{array}{c}5.03 \pm \\
4.50\end{array}$ & 14.50 \\
\cline { 2 - 6 } & $\begin{array}{c}\text { Positive PE } \\
(n=31)\end{array}$ & 2.01 & $\begin{array}{c}35.1 \\
3\end{array}$ & $\begin{array}{c}12.43 \pm \\
10.03\end{array}$ & 33.12 \\
\hline
\end{tabular}

PE: Acute Pulmonary Thromboembolism; CTPA: Computer

Tomography Pulmonary Angiography. SD: Standard deviation

Table 5 shows the descriptive statistics of D-dimer results stratified by Wells criteria risk levels.

4.4.5. D-dimer Test Result Significance for the Outcome of CTPA Findings in the High-risk Group

From the results in the high-risk group (by Wells criteria risk score) we found no correlation between an elevated D-dimer alone and the occurrence of PE, with statistical significance, Fisher's Exact test, $\mathrm{p}=0.630$.

Following guideline recommendations, the use of a $\mathrm{D}$-dimer test in high risk patients is often not recommended; instead the patient should undergo CTPA directly [2], [13]. 
Our data in Table 5 supports such an approach. We have identified the minimum value found in the high-risk group in our studied population to be $1.01 \mathrm{mcg} / \mathrm{mL}$. At a cut of value of $0.50 \mathrm{mcg} / \mathrm{mL}$ these patients would have been considered to have an elevated D-dimer value and therefore, it is likely they would have been sent to CTPA after these results.

\subsubsection{D-dimers Test Result Correlation to Age in Patients 50 Years Old and Older}

The Figure 1 shows the results of a Spearman's correlation coefficient that was computed separately for positive and negative PE by CTPA groups for patients 50 years old or older, aiming to finding a correlation between age and D-dimer test result values. .
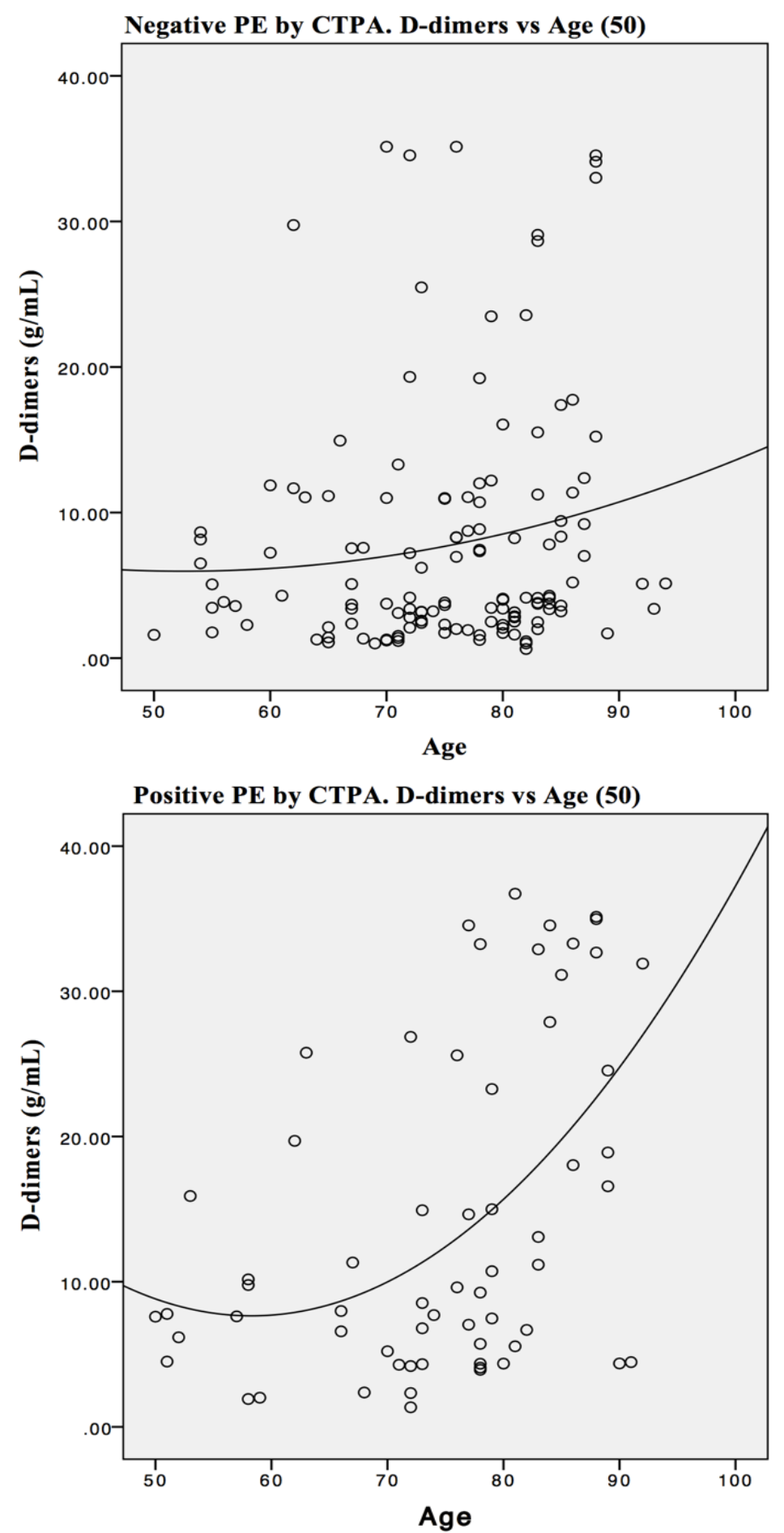

Figure 1. Shows a Spearman's correlation coefficient correlation between d-dimer test value results and patients age ( $\geq 50)$. PE: Acute Pulmonary Thromboembolism; CTPA: Computer Tomography Pulmonary Angiography. 
For the positive PE group, the results show a moderate positive correlation between age and the quantitative value of D-dimer that was found, with statistical significance, rs $=0.425, \mathrm{~N}=67$, (2-tailed), $\mathrm{p}<0.001$.

For the negative PE group, the correlation is only very weak positive with statistical significance, $r s=0.172, \mathrm{~N}=$ 131, (2-tailed), $\mathrm{p}=0.005$.

The elevated D-dimer in the positive group is largely related to the presence of PE in its actual form. In addition, there may be a possible physiologic increase, as well as the likely presence of DVT as part of the PE pathophysiology. The positive but weak correlation between age $(\geq 50$ years old) and increase in D-dimer value seen in patients without $\mathrm{PE}$, can perhaps be explained by the presence of DVT or/and the presence of a physiologic increase in D-dimer.

Of note, several studies have reported a similar positive correlation between $\mathrm{D}$-dimer value and patient age. This may result in a higher number of patients with elevated D-dimer values above the conventional threshold, who ultimately acquire a negative PE result in CTPA [16], [25], [26].

\subsubsection{Age - Adjusted D-dimer Correlation to Age in Patients 50 Years Old and Older}

Following the above mentioned correlation in point 2.3.5. between D-dimer and age, we also tested our data for age adjusted D-dimer from 50 years and older with the previous mentioned formula (1). Adjusting the D-dimer threshold to age resulted in only one case out of a total of one hundred and ninety four cases (1/194) where otherwise considered elevated D-dimer fell under the upper limit threshold. This patient had a negative result for PE in CTPA. No patient with PE had a D-dimer value below the age-adjusted threshold. This may allow theoretical support to implement a threshold for D-dimer results based on age-adjusted D-dimer instead of the standard laboratory threshold. Age-adjusted D-dimer has been recommended by several studies and has shown great efficacy specifically in the exclusion of false positive PE patients [15], [16], [27].

\subsection{Electrocardiography Results for Right Ventricular Dysfunction (RVD) "S1Q3T3" Predictive Value and Discussion}

RVD analysis of ECG resulted in 33/205 (16.1\%) cases with positive ECG S1Q3T3 findings. Of this 23/33 (69.7\%) cases were positive for PE in CTPA. Among the 172/205 $(83.9 \%)$ cases with negative ECG S1Q3T3 findings, $130 / 172(75.6 \%)$ had a negative result for PE in CTPA. S1G3T3 findings in ECG had a sensitivity of $35.38 \%$, a specificity of $92.86 \%$, a positive predictive value of $69.70 \%$, and a negative predictive value of $75.58 \%$ for PE. Previous studies reported S1Q3T3 findings in PE patients to be associated with a worse form of $\mathrm{PE}$ and poor prognosis of a patient. It has also been suggested to be a good predictor when compared to cardiac enzyme markers or brain natriuretic peptide. Additionally, ECG is widely available and inexpensive [28], [29].

This study demonstrates significant specificity for S1Q3T3 findings when a diagnosis of PE is suspected. Given that this finding is associated with more severe forms of PE, we recommend its use in light of its positive predictive value. However, while we can recommend ECG findings to rule out $\mathrm{PE}$, we leave it open to discussion and further study. Furthermore, for patients entering the ED with an ECG performed in the ambulance with concurrent syncope, hypotension or other signs and symptoms of more severe forms of PE; we believe that the ECG findings S1Q3T3 to be of great value in influencing the subsequent actions of the physician.

\section{Conclusions}

1. Analysis of our data revealed increased significance for some clinical presentations. Dyspnea and chest pain were found to be the most relevant clinical findings outside of Wells criteria.

2. This study demonstrates that the data, when tested according to international guidelines and with respective comparison to similar studies, holds value in informing the development of our current approach to $\mathrm{PE}$ diagnosis.

3. The use of a Wells criteria pre-test probability tool and an age-adjusted D-dimer test has been shown to be pertinent in the process of optimizing the diagnostic process in PE.

In patients with a high risk pretest probability, a CTPA is indicated, without prior D-dimer testing. It is believed that this avoids excessive D-dimer testing and shortens the time to diagnosis. Conversely, for those patients with low risk pre-test probability by Wells criteria, our data indicates that there is scope to reduce the number of CTPA examinations performed in this patient group.

This is supported by the evaluation of Wells criteria in our study which highlights the positive correlation between higher risk levels and the prevalence of a positive PE result.

Age-adjusted D-dimer evaluation supports its use over the traditional laboratory cutoff of $0.50 \mathrm{mcg} / \mathrm{mL}$, however this approach should be prospectively evaluated.

An approach including Wells criteria and age-adjusted D-dimer cutoff test may avoid unnecessary steps and examinations.

The clinical relevance of electrocardiogram S1Q3T3 positive findings demonstrated by this study is replicated in several earlier studies. We believe there is a growing pool of evidence justifying the inclusion of these readily-available investigation in future diagnostic approaches. Ultimately, due to its positive predictive value, future evaluation is recommended. 


\section{REFERENCES}

[1] J. A. Heit, "The Epidemiology of Venous Thromboembolism in the Community," Arterioscler. Thromb. Vasc. Biol., vol. 28, no. 3, pp. 370-372, Mar. 2008.

[2] A. F. Members et al., "2014 ESC Guidelines on the diagnosis and management of acute pulmonary embolism," Eur. Heart J., vol. 35, no. 43, pp. 3033-3073, Nov. 2014.

[3] A. T. Cohen et al., "Venous thromboembolism (VTE) in Europe. The number of VTE events and associated morbidity and mortality," Thromb. Haemost., vol. 98, no. 4, pp. 756-764, Oct. 2007.

[4] ISTH Steering Committee for World Thrombosis Day, "Thrombosis: a major contributor to the global disease burden," J. Thromb. Haemost., vol. 12, no. 10, pp. 1580 1590, Oct. 2014.

[5] J. E. Dalen, "Pulmonary Embolism: What have We Learned Since Virchow? Natural History, Pathophysiology, and Diagnosis," Chest, vol. 122, no. 4, pp. 1440-1456, Oct. 2002 .

[6] C. N. Bagot and R. Arya, "Virchow and his triad: a question of attribution," Br. J. Haematol., vol. 143, no. 2, pp. 180190, Oct. 2008.

[7] P. D. Stein et al., "Clinical Characteristics of Patients with Acute Pulmonary Embolism," Am. J. Med., vol. 120, no. 10, pp. 871-879, Oct. 2007.

[8] J. Lucena et al., "Pulmonary embolism and suddenunexpected death: Prospective study on 2477 forensic autopsies performed at the Institute of Legal Medicine in Seville," J. Forensic Leg. Med., vol. 16, no. 4, pp. 196-201, May 2009.

[9] A. Mora, "Invited Commentary: The masquerading pulmonary embolism: why a high index of suspicion remains even today," Proc. Bayl. Univ. Med. Cent., vol. 28, no. 1, p. 71, Jan. 2015.

[10] N. van Es, S. M. Bleker, and M. Di Nisio, "Cancer-associated unsuspected pulmonary embolism," Thromb. Res., vol. 133, pp. S172-S178, May 2014.

[11] C. R. Weiss, E. F. Haponik, G. B. Diette, B. Merriman, J. C. Scatarige, and E. K. Fishman, "Pretest Risk Assessment in Suspected Acute Pulmonary Embolism," Acad. Radiol., vol. 15 , no. 1, pp. 3-14, Jan. 2008.

[12] A. van Belle et al., "Effectiveness of managing suspected pulmonary embolism using an algorithm combining clinical probability, D-dimer testing, and computed tomography," $J A M A$, vol. 295, no. 2, pp. 172-179, Jan. 2006.

[13] A. S. Raja et al., "Evaluation of Patients with Suspected Acute Pulmonary Embolism: Best Practice Advice from the Clinical Guidelines Committee of the American College of Physicians," Ann. Intern. Med., vol. 163, no. 9, pp. 701-711, Nov. 2015

[14] G. Der Sahakian, Y. E. Claessens, J. C. Allo, J. Kansao, G. Kierzek, and J. L. Pourriat, "Accuracy of D-Dimers to Rule Out Venous Thromboembolism Events across Age
Categories," Emerg. Med. Int., vol. 2010, p. e185453, Jul. 2010 .

[15] A. Penaloza et al., "Performance of age-adjusted D-dimer cut-off to rule out pulmonary embolism," J. Thromb. Haemost. JTH, vol. 10, no. 7, pp. 1291-1296, Jul. 2012.

[16] H. J. Schouten et al., "Diagnostic accuracy of conventional or age adjusted D-dimer cut-off values in older patients with suspected venous thromboembolism: systematic review and meta-analysis," The BMJ, vol. 346, May 2013.

[17] I. Co, W. Eilbert, and T. Chiganos, "New Electrocardiographic Changes in Patients Diagnosed with Pulmonary Embolism," J. Emerg. Med.

[18] M. Miniati, C. Cenci, S. Monti, and D. Poli, "Clinical Presentation of Acute Pulmonary Embolism: Survey of 800 Cases," PLOS ONE, vol. 7, no. 2, p. e30891, Feb. 2012.

[19] J. A. Kline, J. Hernandez-Nino, C. D. Newgard, D. N. Cowles, R. E. Jackson, and D. M. Courtney, "Use of pulse oximetry to predict in-hospital complications in normotensive patients with pulmonary embolism," Am. J. Med., vol. 115, no. 3, pp. 203-208, Aug. 2003.

[20] M. L. Posadas-Martínez, F. J. Vázquez, D. H. Giunta, G. D. Waisman, F. G. B. de Quirós, and E. Gándara, "Performance of the Wells score in patients with suspected pulmonary embolism during hospitalization: A delayed-type cross sectional study in a community hospital," Thromb. Res., vol 133, no. 2, pp. 177-181, Feb. 2014.

[21] G. J. Caine, P. S. Stonelake, G. Y. Lip, and S. T. Kehoe, "The Hypercoagulable State of Malignancy: Pathogenesis and Current Debate," Neoplasia N. Y. N, vol. 4, no. 6, pp. 465-473, Nov. 2002.

[22] A. Penaloza, C. Melot, and S. Motte, "Comparison of the Wells score with the simplified revised Geneva score for assessing pretest probability of pulmonary embolism," Thromb. Res., vol. 127, no. 2, pp. 81-84, Feb. 2011.

[23] Z. Y. T. Ong, H. Z. Chai, C. H. How, J. Koh, and T. B. Low, "A simplified approach to haemoptysis," Singapore Med. J., vol. 57, no. 8, pp. 415-418, Aug. 2016.

[24] D. D. Wong, G. Ramaseshan, and R. M. Mendelson, "Comparison of the Wells and Revised Geneva Scores for the diagnosis of pulmonary embolism: an Australian experience," Intern. Med. J., vol. 41, no. 3, pp. 258-263, Mar. 2011

[25] P. L. Harper, E. Theakston, J. Ahmed, and P. Ockelford, "D-dimer concentration increases with age reducing the clinical value of the D-dimer assay in the elderly," Intern. Med. J., vol. 37, no. 9, pp. 607-613, Sep. 2007.

[26] M. Righini, G. Le Gal, A. Perrier, and H. Bounameaux, "The challenge of diagnosing pulmonary embolism in elderly patients: influence of age on commonly used diagnostic tests and strategies," J. Am. Geriatr. Soc., vol. 53, no. 6, pp. 1039-1045, Jun. 2005.

[27] K. Urban, K. Kirley, and J. J. Stevermer, "PURLs: It's time to use an age-based approach to D-dimer," J. Fam. Pract., vol. 63 , no. 3, pp. 155-158, Mar. 2014

[28] S. E. Kim et al., "The Best Predictor for Right Ventricular Dysfunction in Acute Pulmonary Embolism: Comparison between Electrocardiography and Biomarkers," Korean 
Circ. J., vol. 39, no. 9, pp. 378-381, Sep. 2009.

[29] E. Abarca, A. Baddi, and R. Manrique, "ECG manifestations in submassive and massive pulmonary embolism. Report of 4 cases and review of literature," J. Electrocardiol., vol. 47, no. 1, pp. 75-79, Jan. 2014. 